\title{
Metastasiertes Nierenzellkarzinom Sunitinib gilt für das Gros der Patienten als erste Wahl
}

Mit der Einführung von zielgerichteten Therapeutika wie dem Multi-Kinase-Inhibitor Sunitinib (Sutent ${ }^{\circledR}$ ) konnten die Überlebenschancen von Patienten mit fortgeschrittenem Nierenzellkarzinom (mRCC) erstmals deutlich verbessert werden.

In der Phase-III-Zulassungsstudie für Sunitinib waren die Ansprechrate unter dem Multi-Kinase-Inhibitor 4-fach höher als unter IFN- $\alpha$ ( 47 vs. $12 \%)$. Auch das progressionsfreies Überleben (11,5 vs. 5,1 Monate) und das Gesamtüberlebens(26,4 vs. 21,8 Monate) waren signifikant besser. Die nun abgeschlossene Auswertung der Lebensqualitätsdaten belegt, dass Patienten unter Sunitinib zu jedem Zeitpunkt der Therapie auch eine signifikant bessere Lebensqua- lität aufweisen als unter der IFN- $\alpha$-Therapie, so Stefan Siemer, Homburg/Saar.

Aufgrund der jetzt vorliegenden Datenlage wird Sunitinib bei mRCC-Patienten mit niedrigem bis mittlerem Progressionsrisiko - das sind 80-90\% der Patienten - als Therapie der ersten Wahl angesehen, so der Urologe. Dies spiegele sich auch im Therapiealgorithmus der Interdisziplinären Arbeitsgruppe Nierenzellkarzinom der Deutschen Krebsgesellschaft wider. Die ebenfalls zugelassene Kombination von IFN- $\alpha$ und Bevazizumab bietet bei höherer Toxizität und geringerer Praktikabilität geringe Überlebenschancen im Vergleich zu Sunitinib.Dennoch bleiben viele Frage offen, so die Experten. Dies gelte vor allem für die Verbesserung der Zweitlinientherapie und die optimale Abfolge der Medikamente. Auch der mögliche Nutzen einer (neo-)adjuvanten Therapie wird zur Zeit in Studien untersucht.

MW

Quelle: Satelliten-Symposium auf dem 61. Kongress der Deutschen Gesellschaft für Urologie am 17. September 2009 in Dresden; Veranstalter: Pfizer

\section{Knochenmetastasen Brustkrebspatientinnen profitieren von Denosumab}

\begin{abstract}
Viele Krebspatienten entwickeln im Laufe der Erkrankung skelettale Metastasen. Eine vielversprechende Option bietet der humane monoklonale Antikörper Denosumab.
\end{abstract}

Ein zentraler Mediator der ossären Destruktion bei metastasierenden Tumoren ist der endogene Osteoklasten-Aktivator RANKL (Receptor Activator of NF-Kappa B Ligand). Normalerweise wird RANKL von Osteoprotegerin neutralisiert, so Ali- son T. Stopeck, Tucson, AZ/ USA. Bei Knochenmetastasen jedoch dominiert RANKL. Denosumab fängt RANKL $a b$ und verhindert so dessen Bindung an den Rezeptor. In einer Head-toHead-Studie der Phase III wurde Denosumab bei 2.046 Frauen mit

Kurz notiert

GIST und Pankreas-Inselzelltumoren: Sunitinib verlängert das Überleben Bei fortgeschrittenen, nicht resezierbaren und/ oder metastasierten gastrointestinalen Stromatumoren (GIST) bietet Sunitinib (Sutent ${ }^{\circledR}$ ) nach Versagen der Standardtherapie Option: Aktuellen Studien zufolge verlängert der orale MultiKinase-Inhibitor das Intervall bis zur Progression im Mittel um über 40 Wochen, das Gesamtüberleben um 75 Wochen. Wie eine randomisierte internationale Studie zeigt, profitieren auch Pa- tienten mit fortgeschrittenem neuroendokrinem Pankreas-Inselzelltumor von Sunitinib, das bei innen as mittlere progressionsfreie Überleben von 5,5 auf 11 Monate verdoppelte, wie Eric Raymond, Clichy, berichtete auf einem Pressegespräch von Pfizer Oncology im Rahmen des 11th World Congress on Gastrointestinal Cancer in Barcelona. Die häufigsten Nebenwirkungen waren Neutropenie, Hypertonie, gastrointestinale Symptome, Hypoglykämie und Hand-Fuß-Syndrom. fortgeschrittenem Brustkrebs mit Zoledronat verglichen. Die Patientinnen erhielten entweder $120 \mathrm{mg}$ Denosumab s.c. und Placebo i.v. alle 4 Wochen oder $4 \mathrm{mg}$ Zoledronat i.v. und Placebo s.c. alle 4 Wochen. Das Ergebnis: Der Antikörper verzögerte das Auftreten skelettbezogener Ereignisse (SRE) signifikant (HR 0,82; 95 \% KI: 0,71, 0,95). Moderte und schwere Schmerzen im Denosumab-Arm später auf ( 88 vs. 64 Tage $\mathrm{p}=0,009)$. Es kam auch seltener zu Nierenversagen und Akut-Phase-Reaktionen. Nach Stopeck ist der Antikörper eine Therapieoption für das Management von Knochenmetastasen ohne die Notwendigkeit eines renalen Monitorings.

Quelle: Amgen Pivotal Data Press Briefing Im Rahmen des ESMO/ECCO in Berlin am 22. September 2009 